\title{
Parental Separation and Semen Quality in Young Men: A Population-Based Cohort Study
}

\begin{abstract}
Anne Gaml-Sørensen $\mathbb{D}^{1}$, Nis Brix $\mathbb{D}^{1,2}$, Gunnar Toft ${ }^{3,4}$, Tine Brink Henriksen $\mathbb{D}^{5}$, Andreas Ernst ${ }^{1,6}$, Linn Håkonsen Arendt ${ }^{1,7}$, Sandra Søgaard Tøttenborg $\mathbb{D}^{8}$, Katia Keglberg Hærvig, ${ }^{8,9}$, Karin Sørig Hougaard (D) ${ }^{9,10}$, Jens Peter Ellekilde Bonde ${ }^{8,9}$, Cecilia Høst Ramlau-Hansen (D)

'Department of Public Health, Research Unit for Epidemiology, Bartholins Allé 2, Aarhus University, Aarhus C, 8000, Denmark; ${ }^{2}$ Department of Clinical Genetics, Aarhus University Hospital, Aarhus N, 8200, Denmark; ${ }^{3}$ Department of Clinical Epidemiology, Aarhus University, Aarhus N, 8200, Denmark; ${ }^{4}$ Steno Diabetes Center Aarhus, Aarhus University Hospital, Aarhus N, 8200, Denmark; ${ }^{5}$ Perinatal Epidemiology Research Unit, Department of Paediatrics and Adolescent Medicine, Aarhus University Hospital, Aarhus N, 8200, Denmark; ${ }^{6}$ Department of Urology, Aarhus University Hospital, Aarhus N, 8200, Denmark; ${ }^{7}$ Department of Obstetrics and Gynecology, Aarhus University Hospital, Aarhus N, 8200, Denmark; ${ }^{8}$ Department of Occupational and Environmental Medicine, Bispebjerg and Frederiksberg Hospital, Copenhagen NV, 2400, Denmark; ${ }^{9}$ Department of Public Health, University of Copenhagen, København K, 1014, Denmark; ${ }^{10}$ National Research Centre for the Working Environment, Copenhagen East, 2100 , Denmark
\end{abstract}

Correspondence: Anne Gaml-Sørensen, Department of Public Health, Research Unit for Epidemiology, Bartholins Allé 2, Aarhus University, Aarhus C, 8000, Denmark, Tel +45 40868I83 Email ags@ph.au.dk

Purpose: Parental separation may be a stressful life event with the potential to influence hormonal regulation of offspring reproductive health and thereby affect semen quality in young men. We aimed to study the association between parental separation in pregnancy or in childhood and semen quality in young men and to study whether the timing of parental separation in childhood was important.

Patients and Methods: We conducted a follow-up study of 1058 young men born 1998-2000 from the Fetal Programming of Semen Quality (FEPOS) cohort nested within the Danish National Birth Cohort. Data on parental separation were obtained longitudinal by self-report. Parental separation in pregnancy was dichotomized, and parental separation in childhood was both dichotomized and categorized according to the timing of parental separation (from birth, from early childhood ( $0-5$ years), and from late childhood (6-10 years)). Semen volume, concentration, total sperm count, motility, morphology, and testes volume were analysed using multivariable negative binomial regression models.

Results: Parental separation in pregnancy was not associated with semen quality. The association between parental separation in childhood and semen quality differed with the timing of parental separation. Parental separation from birth was associated with higher semen volume of $25 \%, 95 \%$ CI $(-5 ; 64)$; higher total sperm count of $62 \%, 95 \%$ CI $(-6 ; 179)$; and higher proportion of morphologically normal spermatozoa of $59 \%, 95 \%$ CI $(20 ; 111)$. Parental separation in early childhood was associated with lower semen volume of $-14 \%, 95 \%$ CI $(-24 ;-3)$; lower concentration of $-15 \%, 95 \%$ CI $(-28 ; 1)$; lower total sperm count of $-17 \%, 95 \%$ CI $(-32 ; 2)$ and lower testes volume of $-11 \%, 95 \%$ CI $(-18 ;-3)$.

Conclusion: The timing of parental separation was important, and parental separation from birth was associated with higher semen quality, and parental separation in early childhood was associated with lower semen quality.

Keywords: stressful life-events, parental divorce, semen analysis, sperm quality, male infertility, epidemiology

\section{Introduction}

Poor male reproductive health is a concern in developed countries, ${ }^{1-3}$ and a prenatal origin has been suggested. ${ }^{2-4}$ Prenatal factors can interfere with intrauterine reproductive organ development and development and maturation of the hypothalamic-pituitary-gonadal (HPG) axis, which could possibly affect semen quality in the young men.,

Parental separation in pregnancy, or single motherhood, may induce maternal stress. ${ }^{7}$ Maternal stress has been suggested to introduce epigenetic changes in fetal DNA, to affect the hypothalamic-pituitary-adrenal (HPA) axis ${ }^{8}$ and to influence the fetal hormonal environment. ${ }^{9}$ This may potentially disturb reproductive health in male offspring. ${ }^{9,10}$ 
Stress due to bereavement ${ }^{11}$ and other stressful life events in pregnancy ${ }^{12}$ has been associated with poor male reproductive health; however, parental separation in pregnancy has not been studied yet.

Parental separation in childhood may be a major stressful life event for all family members, including the children. ${ }^{13-15}$ Research highlights the importance of addressing the timing of parental separation ${ }^{16}$ and in general, preschool children may be more susceptible towards stress following parental separation. ${ }^{17,18}$ Long-term consequences may arise due to hormonal changes and elevated cortisol levels following chronic augmentation of the HPA axis. ${ }^{19,20}$ This increased HPA axis activity may in turn suppress the HPG axis, since these interact, ${ }^{21}$ and may thereby influence semen quality due to its effect on spermatogenesis. ${ }^{10}$

The experience of stressful life events in childhood have been suggested to be associated with low semen quality ${ }^{22}$; however, parental separation has not been studied yet. In Denmark and in other Western countries, parental separation affects up to $50 \%$ of all children, ${ }^{23}$ highlighting the need to investigate potential long-term consequences hereof. We aimed to investigate whether parental separation in pregnancy or in childhood was associated with semen quality in young men from a large population-based cohort, and whether the timing of parental separation in childhood was important. We hypothesized that parental separation is associated with lower semen quality.

\section{Materials and Methods}

This cohort study is based on the Fetal Programming of Semen Quality (FEPOS) cohort, ${ }^{24}$ a sub-cohort within the Danish National Birth Cohort (DNBC). ${ }^{25}$

In the DNBC, pregnant women were invited to provide information on health behavior and medical history in the first trimester through a computer-assisted telephone interview. A follow-up questionnaire for the child to complete under parental guidance was mailed to the mothers, when the child was 11 years. ${ }^{25}$ The questionnaires can be found at https:// www.dnbc.dk/data-available.

Young men were eligible for invitation to the FEPOS cohort, if their mothers had completed the first and second interviews in the DNBC and had a gestational blood sample available in the Danish national biobank. The men were eligible if they were at least 18 years and 9 months old and lived in the area of Aarhus or Copenhagen. During the recruitment period from March 2017 to December 2019, a total of 21,623 men were eligible for participation. Of those, 5,697 men were randomly and consecutively invited to participate using their personal and secure digital mailbox "e-Boks" (Figure 1). The men were encouraged to decline participation, if they had only one or no testes in the scrotum, or if they had undergone sterilization, orchidectomy, or chemotherapy. After providing informed consent, the men completed an online questionnaire on health behavior and medical history, participated in a clinical examination, and provided a semen sample. In total, 1,058 men participated (19\%). A detailed description of the recruitment and inclusion can be found in the cohort profile. ${ }^{24}$

\section{Parental Separation}

Information on parental separation in pregnancy was obtained from the first trimester interview within the DNBC. The pregnant women were asked, if they had a cohabiting spouse or partner. Cohabiting women were assigned to the unexposed group, and non-cohabiting women to the exposure group of parental separation in pregnancy.

Information on parental separation in childhood was obtained from the 11-year follow-up questionnaire completed by a subset $(n=702(66 \%))$ of the children within the FEPOS cohort. The children were asked to report on parental cohabitation, including current and previous cohabitation status of their parents and their own age at parental separation. We excluded children experiencing death of a parent $(n=6)$ to avoid pooling of stress due to parental separation with bereavement due to death of a parent. ${ }^{15}$ Children living with both parents until 11 years of age were assigned to the reference category of no parental separation. For the dichotomous exposure categorization, all other children were assigned to the exposure category of parental separation in childhood.

To address the timing of parental separation in relation to the specific health consequences under investigation, ${ }^{15,16}$ we further categorized the exposure category of parental separation in childhood according to what was previously seen for parental separation relative to timing of pubertal development. ${ }^{26}$ If the children stated that their parents never lived together, they were assigned to the exposure category parental separation from birth. If they stated that their parents 


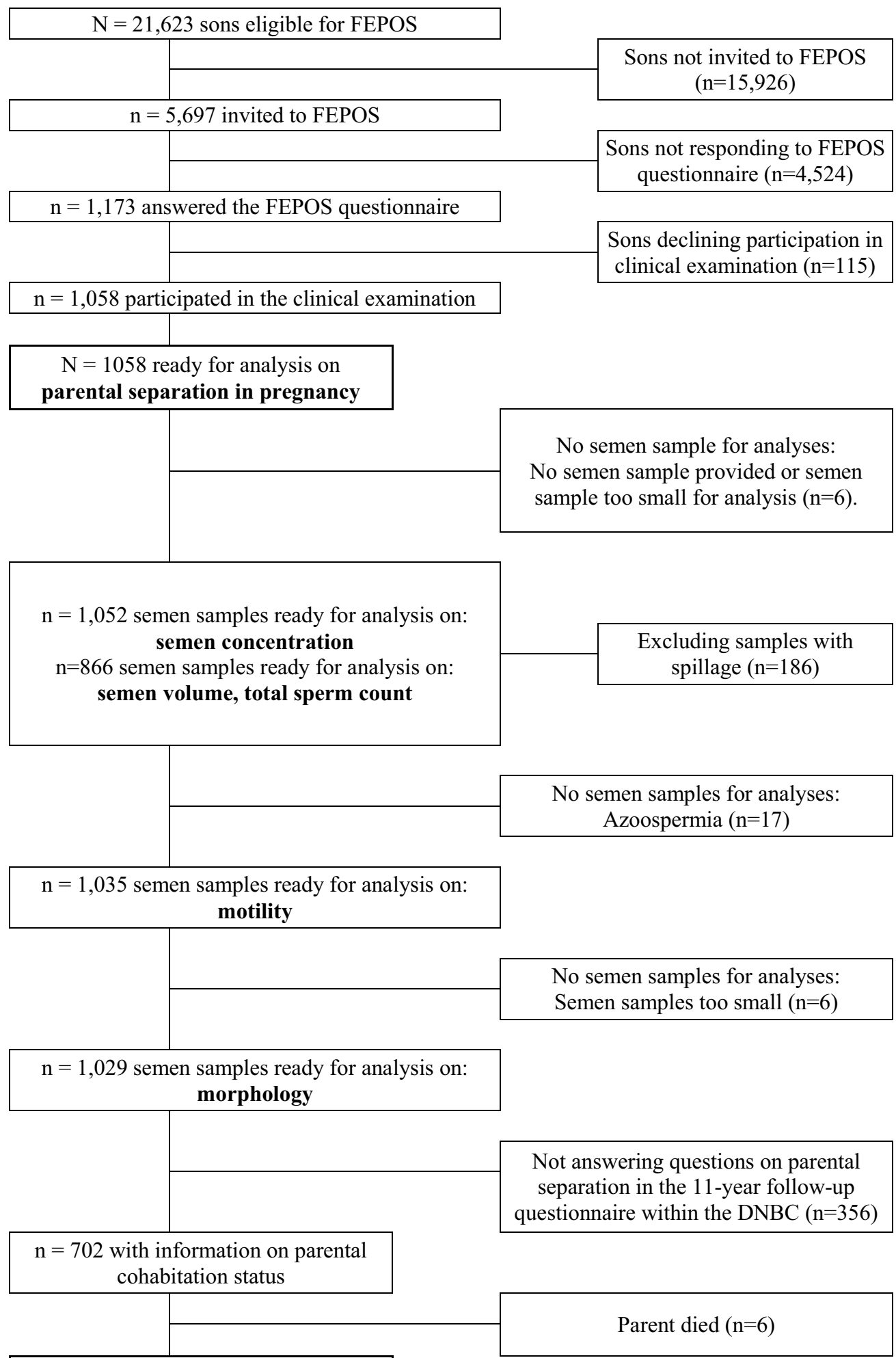

$\mathrm{N}=696$ ready for analysis on parental separation in childhood

Figure I Flowchart on the inclusion of participants in the fetal programming of semen quality (FEPOS) cohort, nested within the Danish National Birth Cohort, Denmark, 1998-2019. 
separated between the ages of $0-5$ years, the children were assigned to the exposure category parental separation in early childhood. If they stated that their parents separated between the ages of 6-10 years, the children were assigned to the exposure category parental separation in late childhood.

It was not possible to combine the categorized parental separation in childhood exposure groups with the parental separation in pregnancy exposure group, due to incomplete overlap. Some participants reporting parental separation in pregnancy did not have available data on parental separation in childhood $(n=7(32 \%))$, and some did not report parental separation from birth $(n=5(23 \%))$, potentially reflecting that the parents might have moved in with each other between the pregnancy interview and the birth. Therefore, parental separation in pregnancy and in childhood were investigated as two separate exposures (Figure 1).

\section{Semen Quality and Testes Volume}

Semen samples were collected at home or at the research facility in a pre-weighed sample container. Prior to sample collection, participants were instructed to be abstinent for at least 48-72 hours. All semen quality measures were analysed according to recommendations from World Health Organization. ${ }^{27}$ All measures were quality controlled and met given standards for semen quality measures. ${ }^{24}$

Semen volume was estimated by weighing of the sample. Motility and sperm concentration were assessed manually in duplicates after liquefaction, by one of two experienced biomedical laboratory technicians in Aarhus or Copenhagen. Concentration was calculated after standard sperm dilution and counting. Motility was assessed as proportions of progressive (PR), non-progressive (NP) and immotile (IM) spermatozoa. In the statistical analyses, we modelled NP + IM in percentage to ensure optimal model fit. Total sperm count was calculated by multiplying concentration and volume. Morphology, measured as the proportion of strictly normal spermatozoa, was analysed at the Centre of Reproductive Medicine in Malmö, Sweden. At least 200 spermatozoa were assessed in each ejaculate, and abnormalities were recorded for all four regions of the spermatozoa. ${ }^{24}$ Testes volume was self-assessed by the young men using a Prader Orchidometer, which previously have been found to be valid. ${ }^{28}$

\section{Covariates}

All covariates were identified a priori by use of existing literature and Directed Acyclic Graphs. ${ }^{29}$ We included maternal socioeconomic status, first trimester smoking, and age at delivery. In our sub-analyses, we further included information on unplanned pregnancy (yes, no). Due to marked collinearity with the exposure categories, this variable was not included in the main analyses. Age at delivery was obtained from the Danish Medical Birth Registry, a nationwide register that holds information on all births in Denmark. ${ }^{30}$ Information on unplanned pregnancy and first trimester smoking was obtained from the DNBC first trimester interview, as was socioeconomic status, which was defined according to occupation and level of education derived from the International Standard Class of Occupation and Education codes (ISCO-88 and ISCED).

Variables expected to be strongly associated with semen quality were also included in the multivariable models to improve precision. ${ }^{31}$ We included place of semen sample collection (at home or the clinic), interval from ejaculation to motility analysis (in minutes), spillage of semen sample (yes, no), and abstinence time (in days). Information on all precision variables were obtained at the clinical examination. As we were interested in the total effect of parental separation on semen quality and testicular volume, we did not include sons' own health behavior in the analyses, as this was considered potential mediating factors.

\section{Statistical Analysis}

Baseline characteristics were reported as numbers and percentages or means and standard deviations (SD) according to parental separation in pregnancy and to parental separation in childhood. Semen quality characteristics were reported as 10th, 50th and 90th pseudo percentiles, also according to categories of parental separation. Pseudo percentiles were calculated as the mean of the five values nearest to the actual percentile to comply with local regulations (GDPR, Regulation (EU), 2016/679 of 25 May 2018), which state that a single value corresponding to a single participant may not be reported. 
The negative binomial distribution fitted data well compared to other distributions and transformations. Therefore, the data was analysed using a multivariable negative binomial regression model fitted by maximum likelihood estimation (STATA's -nbreg- package). Crude and adjusted mean ratios of semen quality measures according to exposure groups were estimated with $95 \%$ confidence intervals (CI). Based on these ratios, relative percentage differences were calculated as follows: (ratio -1$) \times 100 \%$.

All models were adjusted for the selected maternal characteristics and precision variables. Since information on potential confounding variables was available for all participants, complete case analyses were conducted. Maternal age and abstinence time were modelled as second-order polynomials to allow for non-linearity. ${ }^{32}$ Participants, who reported spillage during sample collection $(\mathrm{n}=186)$, were excluded from the models examining volume and total sperm count. In the remaining models, spillage (yes, no) was adjusted for. Time from ejaculation to motility analysis was applied only in models examining motility.

Selection weights were applied to account for potential selection bias due to non-participation. ${ }^{33}$ In short, a logistic regression model was fitted with participation as the dependent variable and parental separation in addition to the selected covariates potentially associated with participation as explanatory variables. The inverse of the model-based predicted probability of participation was used as the selection weight in all models. See Supplementary Material 1 for a detailed description. To account for the use of selection weights and clustering of siblings, robust standard errors were applied in all analyses.

The negative binomial regression models were checked by comparing the observed distributions against the modelbased distributions using Q-Q plots. Then, standardized deviance residuals were plotted against model-based predictions. The data check did not raise concerns about model fit (data not shown). Data management and statistical analyses were conducted in STATA 16.1 (StataCorp, College Station, TX).

\section{Ethics}

This study was conducted in accordance with the Declaration of Helsinki. All participants provided a written informed consent at enrolment. The Committee for Biomedical Research Ethics in Denmark has approved data collection in the DNBC ((KF) 01-471/94). The establishment of the FEPOS cohort was approved by the Scientific Research Ethics Committee for Copenhagen and Frederiksberg (No. H-16015857) and the Danish Data Protection Agency (P-2019-503). The data collection was approved by the Danish Data Protection Agency (2012-41-0379 and 2015-57-0002) and the Steering Committee of the DNBC (Ref. no. 2016-08). This particular study was approved by the Steering Committee of the DNBC (Ref. no. 2019-21).

\section{Results}

Of the 1,058 FEPOS participants, 22 men (2\%) were exposed to parental separation in pregnancy (Table 1), while 160 men (23\%) were exposed to parental separation in childhood (Table 2). Mothers of men exposed to separation in pregnancy or in childhood were more likely to report smoking and to have lower socioeconomic status in the first trimester. The participants had a median age of 19 years and 2 months (range 18 years and 10 months to 21 years and 4 months). Among non-participants, $2 \%$ were exposed to parental separation in pregnancy, and $22 \%$ were exposed to parental separation in childhood (data not in table).

\section{Parental Separation in Pregnancy}

Parental separation in pregnancy was not associated with semen quality (Table 3, Figure 2 and Supplemental Table 1). Adjusted associations pointed towards both higher (eg, semen volume of 13\%, 95\% CI $(-6 ; 36)$ ) and lower (eg, semen concentration of $-13 \%, 95 \%$ CI $(-42,30))$ semen quality in exposed compared to unexposed men.

\section{Parental Separation in Childhood}

The dichotomized parental separation in childhood was associated with a tendency towards lower semen quality in exposed men (Table 3), but all confidence intervals included the null (Figure 2 and Supplemental Table 1). For example, exposed men had lower semen concentration of $-12 \%, 95 \%$ CI $(-25 ; 4)$; and lower total sperm count of $-10 \%, 95 \%$ CI $(-25 ; 8)$ compared to unexposed men. 
Table I Baseline Characteristics. Maternal and Semen Quality Characteristics According to Parental Separation in Pregnancy Among 1058 Young Men from FEPOS, Denmark, 1998-2019

\begin{tabular}{|c|c|c|c|c|c|c|}
\hline & \multicolumn{4}{|c|}{ Parental Separation } & \multicolumn{2}{|c|}{ Missing Values } \\
\hline & \multicolumn{2}{|c|}{ Yes } & \multicolumn{2}{|c|}{ No } & \multirow[b]{2}{*}{ No. } & \multirow[b]{2}{*}{$\%$} \\
\hline & No. & $\%$ & No. & $\%$ & & \\
\hline & 22 & 2.1 & 1,036 & 97.9 & & \\
\hline \multicolumn{7}{|l|}{ Maternal characteristics } \\
\hline Maternal age at delivery; mean years (SD) & \multicolumn{2}{|c|}{$31.5(4.8)$} & \multicolumn{2}{|c|}{$30.5(4.1)$} & $<5^{\mathrm{a}}$ & 0.5 \\
\hline Maternal social class; n(\%) & & & & & 0 & 0.0 \\
\hline High-/low-grade professional & 5 & 22.7 & 704 & 68.0 & & \\
\hline Skilled/unskilled worker & 8 & 36.4 & 292 & 28.2 & & \\
\hline Student/economically inactive & 9 & 40.9 & 40 & 3.8 & & \\
\hline Smoking I. trimester; $n(\%)$ & & & & & 0 & 0.0 \\
\hline 0 cigarettes/day & 7 & 31.8 & 808 & 78.0 & & \\
\hline I-10 cigarettes/day & $>10$ & $>45.5$ & 194 & 18.7 & & \\
\hline$>10$ cigarettes/day & $<5^{\mathrm{a}}$ & $<22.7$ & 34 & 3.28 & & \\
\hline Unplanned pregnancy; n(\%) & & & & & 6 & 0.6 \\
\hline No & 6 & 27.3 & 871 & 84.1 & & \\
\hline Yes & 16 & 72.7 & 159 & 15.4 & & \\
\hline \multicolumn{7}{|l|}{ Precision variables } \\
\hline Abstinence time; mean days (SD) & \multicolumn{2}{|c|}{$2.1(0.9)$} & \multicolumn{2}{|c|}{$2.3(1.5)$} & \multirow[t]{3}{*}{5} & \multirow[t]{3}{*}{0.5} \\
\hline $0-2$ days; $n(\%)$ & 7 & 31.8 & 359 & 34.7 & & \\
\hline$>2$ days; $n(\%)$ & 15 & 68.2 & 672 & 64.9 & & \\
\hline Place of semen sample collection; $n(\%)$ & & & & & 10 & 1.0 \\
\hline At home & $<5^{\mathrm{a}}$ & $<22.7$ & 134 & 12.9 & & \\
\hline In the clinic & $>17$ & $>77.3$ & 892 & 86.1 & & \\
\hline Spillage; n(\%) & & & & & 9 & 0.9 \\
\hline Yes & $<5^{\mathrm{a}}$ & $<22.7$ & $|8|$ & 17.5 & & \\
\hline No & $>17$ & $>77.3$ & 846 & 81.7 & & \\
\hline Interval ejaculation - analysis; mean min (SD) & \multicolumn{2}{|c|}{$53.0(17.5)$} & \multicolumn{2}{|c|}{$49.9(19.5)$} & 12 & 1.2 \\
\hline 0-60 min; $n(\%)$ & 13 & 59.1 & 773 & 74.6 & & \\
\hline$>60$ min; $n(\%)$ & 9 & 40.9 & 251 & 24.2 & & \\
\hline
\end{tabular}

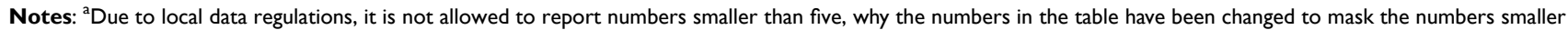
than five.

Abbreviations: SD, standard deviation.

For the subdivision of parental separation in childhood, parental separation from birth was associated with higher semen quality in exposed compared to unexposed men (Table 3); eg higher semen volume of $25 \%, 95 \%$ CI $(-5 ; 64)$; higher total sperm count of $62 \%, 95 \%$ CI $(-6 ; 179)$; and a higher proportion of morphological normal spermatozoa of 59\%, 95\% CI $(20 ; 111)$ (Figure 3 and Supplemental Table 2). In contrast, parental separation in early childhood was associated with lower semen quality (Table 3 ); eg lower semen volume of $-14 \%, 95 \%$ CI $(-24 ;-3)$; lower semen concentration of $-15 \%, 95 \% \mathrm{CI}(-28 ; 1)$; lower total sperm count of $-17 \%, 95 \% \mathrm{CI}(-32 ; 2)$; and lower testes volume of $-11 \%, 95 \%$ CI $(-18 ;-3)$ (Figure 3 and Supplemental Table 2). Parental separation in late childhood was not associated with semen quality (Table 3, Figure 3 and Supplemental Table 2). 
Table 2 Baseline Characteristics. Maternal and Semen Quality Characteristics According to Parental Separation in Childhood Among 696 Young Men from FEPOS, Denmark, 1998-2019

\begin{tabular}{|c|c|c|c|c|c|c|}
\hline & \multicolumn{4}{|c|}{ Parental Separation } & \multicolumn{2}{|c|}{ Missing Values } \\
\hline & \multicolumn{2}{|c|}{ Yes } & \multicolumn{2}{|c|}{ No } & \multirow[b]{2}{*}{ No. } & \multirow[b]{2}{*}{$\%$} \\
\hline & No. & $\%$ & No. & $\%$ & & \\
\hline & 160 & 23.0 & 536 & 77.0 & & \\
\hline \multicolumn{7}{|l|}{ Maternal characteristics } \\
\hline Maternal age at delivery; mean years (SD) & \multicolumn{2}{|c|}{$30(4.4)$} & \multicolumn{2}{|c|}{$30.5(4.0)$} & $<5^{\mathrm{a}}$ & 0.7 \\
\hline Maternal social class; $\mathrm{n}(\%)$ & & & & & 0 & 0.0 \\
\hline High-/low-grade professional & 94 & 60.2 & 371 & 69.2 & & \\
\hline Skilled/unskilled worker & 55 & 33.1 & 139 & 25.9 & & \\
\hline Student/economically inactive & II & 6.6 & 26 & 4.9 & & \\
\hline Smoking I. trimester; n(\%) & & & & & 0 & 0.0 \\
\hline 0 cigarettes/day & 108 & 66.9 & 442 & 82.5 & & \\
\hline I-10 cigarettes/day & 44 & 27.7 & 81 & 15.1 & & \\
\hline$>10$ cigarettes/day & 8 & 5.4 & 13 & 2.4 & & \\
\hline Unplanned pregnancy; n(\%) & & & & & $<5^{\mathrm{a}}$ & 0.7 \\
\hline No & 119 & 74.4 & $<470$ & $<87.7$ & & \\
\hline Yes & $4 I$ & 25.6 & $<66$ & $<12.3$ & & \\
\hline \multicolumn{7}{|l|}{ Precision variables } \\
\hline Abstinence time; mean days (SD) & \multicolumn{2}{|c|}{$2.2(1.2)$} & \multicolumn{2}{|c|}{$2.4(1.6)$} & \multirow[t]{3}{*}{$<5^{\mathrm{a}}$} & \multirow[t]{3}{*}{0.7} \\
\hline 0-2 days; $\mathrm{n}(\%)$ & 59 & 36.9 & 186 & 34.7 & & \\
\hline$>2$ days; $n(\%)$ & $<101$ & $<63.1$ & $<350$ & $<65.3$ & & \\
\hline Place of semen sample collection; $n(\%)$ & & & & & 8 & 1.2 \\
\hline At home & $<28$ & $<17.5$ & $<68$ & $<12.7$ & & \\
\hline In the clinic & 132 & 82.5 & 468 & 87.3 & & \\
\hline Spillage; n(\%) & & & & & 8 & 1.2 \\
\hline Yes & $<26$ & $<16.3$ & $<108$ & $<20.1$ & & \\
\hline No & 134 & 83.8 & 428 & 79.9 & & \\
\hline Interval ejaculation - analysis; mean min (SD) & \multicolumn{2}{|c|}{$51.3(20.7)$} & \multicolumn{2}{|c|}{$50.0(19.4)$} & 6 & 0.9 \\
\hline $0-60 \min ; n(\%)$ & 109 & 68.1 & 407 & 75.9 & & \\
\hline$>60$ min; $n(\%)$ & $<51$ & 31.9 & $<129$ & $<24.1$ & & \\
\hline
\end{tabular}

Notes: a Due to local data regulations, it is not allowed to report numbers smaller than five, why the numbers in the table have been changed to mask the numbers smaller than five.

Abbreviations: SD, standard deviation.

\section{Sub-Analyses}

Overall, the estimates for semen quality did not change essentially with further adjustment for unplanned pregnancy (Supplementary Tables S3 and $\underline{\mathrm{S}}$ ).

\section{Discussion}

Parental separation in pregnancy was not associated with semen quality. Dichotomized parental separation in childhood was associated with a tendency towards lower semen quality, but effect sizes were small and confidence intervals wide and overlapped the null. Parental separation from birth was associated with a tendency towards higher semen quality, whereas parental separation in early childhood was associated with a tendency towards lower semen quality. Though 
Table 3 Descriptive Statistics ${ }^{\mathrm{a}}$. Semen Quality Characteristics and Testes Volume According to (A) Parental Separation in Pregnancy; (B) Parental Separation in Childhood; and (C) Parental Separation According to Timing of Parental Separation in Childhood in Young Men from FEPOS, Denmark, 2017-2019

\begin{tabular}{|c|c|c|c|c|c|c|c|c|c|c|}
\hline \multirow[t]{4}{*}{ A. } & \multicolumn{8}{|c|}{ Parental Separation in Pregnancy $(n=1058)$} & \multirow{2}{*}{\multicolumn{2}{|c|}{ Missing Values }} \\
\hline & \multicolumn{4}{|c|}{ Yes } & \multicolumn{4}{|c|}{ No } & & \\
\hline & \multicolumn{2}{|c|}{ No. } & \multicolumn{2}{|c|}{$\%$} & \multicolumn{2}{|c|}{ No. } & \multicolumn{2}{|c|}{$\%$} & No. & $\%$ \\
\hline & \multicolumn{2}{|c|}{22} & \multicolumn{2}{|c|}{2.1} & \multicolumn{2}{|c|}{1,036} & \multicolumn{2}{|c|}{97.9} & & \\
\hline Volume $(\mathrm{mL})^{\mathrm{b}}$ & \multicolumn{4}{|c|}{$2.9(1.5-4.8)$} & \multicolumn{4}{|c|}{$2.7(1.3-4.7)$} & 192 & 18 \\
\hline Concentration (mill/mL) & \multicolumn{4}{|c|}{$34(6-98)$} & \multicolumn{4}{|c|}{$39(8-113)$} & 6 & 0.6 \\
\hline Total sperm count (mill) ${ }^{\mathrm{b}}$ & \multicolumn{4}{|c|}{$96(20-336)$} & \multicolumn{4}{|c|}{$102(18-338)$} & 192 & 18 \\
\hline Progressive motility $(\mathrm{PR} \%)^{\mathrm{c}}$ & \multicolumn{4}{|c|}{$65(47-77)$} & \multicolumn{4}{|c|}{$63(4 I-80)$} & 23 & 2.2 \\
\hline Morphology $\left(\%\right.$ normal) ${ }^{c}$ & \multicolumn{4}{|c|}{$8.4(1.0-13.4)$} & \multicolumn{4}{|c|}{$6.0(1.0-13.0)$} & 29 & 2.7 \\
\hline Average testicular volume $(\mathrm{mL})$ & \multicolumn{4}{|c|}{$19(8-24)$} & \multicolumn{4}{|c|}{$15(9-24)$} & $<5^{d}$ & 0.5 \\
\hline \multirow[t]{4}{*}{ B. } & \multicolumn{10}{|c|}{ Parental Separation in Childhood $(n=696)$} \\
\hline & \multicolumn{4}{|c|}{ Yes } & & No & & & Missir & alues \\
\hline & & & & & & & & & No. & $\%$ \\
\hline & & & & & & & & & & \\
\hline Volume $(\mathrm{mL})^{\mathrm{b}}$ & & & $4-4.5)$ & & & $2.7(1.3$ & & & 134 & 19 \\
\hline Concentration (mill/mL) & & & -90) & & & $39(8-$ & & & $<5^{d}$ & 0.7 \\
\hline Total sperm count (mill) ${ }^{\mathrm{b}}$ & & & $-324)$ & & & $102(18$ & & & 134 & 19 \\
\hline Progressive motility $\left(a\right.$ \%) ${ }^{c}$ & & & $9-81)$ & & & $62(38$ & & & 14 & 2.0 \\
\hline Morphology $(\% \text { normal })^{c}$ & & & $-13.0)$ & & & $6.0(1.0-$ & & & 18 & 2.6 \\
\hline Average testicular volume $(\mathrm{mL})$ & & & $-24)$ & & & $15(9-$ & & & $<5^{d}$ & 0.5 \\
\hline C. & & & & ental Se & ation in & Idhood & 696) & & & \\
\hline & & & & & & & & & Missir & alues \\
\hline & Fror & irth & n Earl & ildhood & In Late & Ildhood & & & & \\
\hline & No. & $\%$ & No. & $\%$ & No. & $\%$ & No. & $\%$ & No. & $\%$ \\
\hline & 10 & 1.4 & 83 & 11.9 & 67 & 9.6 & 536 & 77 & & \\
\hline Volume $(\mathrm{mL})^{\mathrm{b}}$ & 3.8 & 4.7) & 2.4 & $-4.2)$ & 2.6 & $-4.6)$ & 2.7( & -4.9) & 134 & 19 \\
\hline Concentration (mill/mL) & 40 & 79) & & & & 10) & 39 & 12) & $<5^{d}$ & 0.7 \\
\hline Total sperm count (mill) ${ }^{b}$ & 158 & 300) & 92 & 292) & 96 & 324) & 102 & 349) & 134 & 19 \\
\hline Progressive motility $\left(a\right.$ \%) ${ }^{c}$ & 62 & 68) & & 80) & & 84) & 62 & 79) & 14 & 2.0 \\
\hline Morphology $(\% \text { normal })^{c}$ & 11.0 & -13.4) & 6.0 & 12.5) & 5.5 & |3.0) & $6.0(1$ & 13.0) & 18 & 2.6 \\
\hline Average testicular volume $(\mathrm{mL})$ & 19 & 23) & & & & $-25)$ & 15 & & $<5^{d}$ & 0.5 \\
\hline
\end{tabular}

Notes: ${ }^{a}$ Reported as 50th percentile (10th-90th). All percentiles are pseudo percentiles calculated from the average of five values. ${ }^{b}$ Excluding samples from participants reporting spillage. 'Excluding samples with azoospermia. ${ }^{\mathrm{d} D u e}$ to local data regulations, it is not allowed to report numbers smaller than five. Abbreviations: p10, 10th pseudo percentile; p50, 50th pseudo percentile; p90, 90th pseudo percentile. 


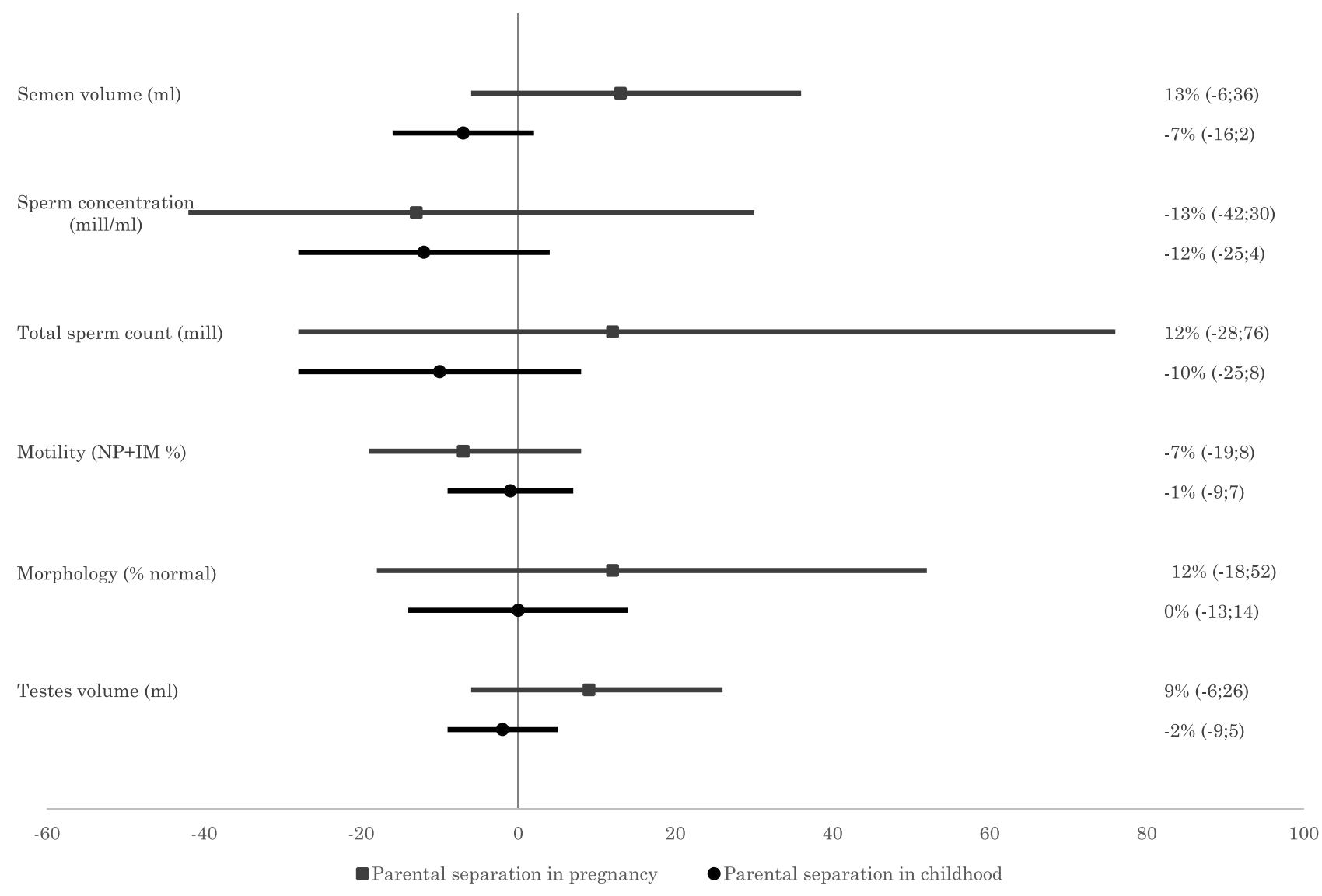

Figure 2 Main results for parental separation in pregnancy or in childhood. Relative percentage differences in semen quality and testes volume according to parental separation in pregnancy (square and dark grey $(\mathrm{N}=\mathrm{I}, 058)$ ) or in childhood (circle and black $(\mathrm{N}=696))$. Parental separation relatively to no parental separation (vertical line). Adjusted for maternal age at delivery; maternal social class; maternal first trimester smoking; abstinence time; place of semen sample collection (not testes volume); spillage (not testes volume). Motility further adjusted for interval between ejaculation and analysis in minutes. Due to model fit, the figure show results for NP (non-progressive) + IM (immotile) spermatozoa. Therefore, negative estimates represent a higher proportion of progressive motility, and positive estimates represent a lower proportion of progressive motility. Point estimates with $95 \%$ confidence intervals are shown to the right.

most confidence intervals included the null, the tendencies were evident for all semen quality characteristics and the lack of statistical significance may be a consequence of few exposed participants and hence low-powered analyses, more than suggestive of null-findings.

\section{Strengths and Weaknesses}

In this population-based follow-up study, we used information on a large sample of young men from a nation-wide birth cohort. ${ }^{24}$ The participation rate was relatively low, introducing risk of selection bias. However, the study population was restricted to young men, presumably unaware of their own fertility status. It is therefore unlikely that their participation was associated with semen quality. ${ }^{34}$ Further, participation was not associated with parental separation, since the proportion of men exposed to parental separation were similar between participants and non-participants. We estimated selection weights to further reduce the risk of selection bias. Hence, the risk of selection bias is considered limited.

Although individual semen samples may vary over time, the within individual variation is not likely to introduce systematic errors, when one semen sample per participant is obtained and analysed. ${ }^{35,36}$ Though self-assessment of testes volume may be valid, some participants may have underestimated their testes volume. ${ }^{28}$ This may, however, not introduce systematic bias, as underestimation are likely independent and non-differential with regard to participants' exposure status. The trained biomedical laboratory technicians were blinded to the participants' exposure, and therefore potential measurement error on semen quality is non-differential according to the exposure groups, potentially attenuating the observed associations. 


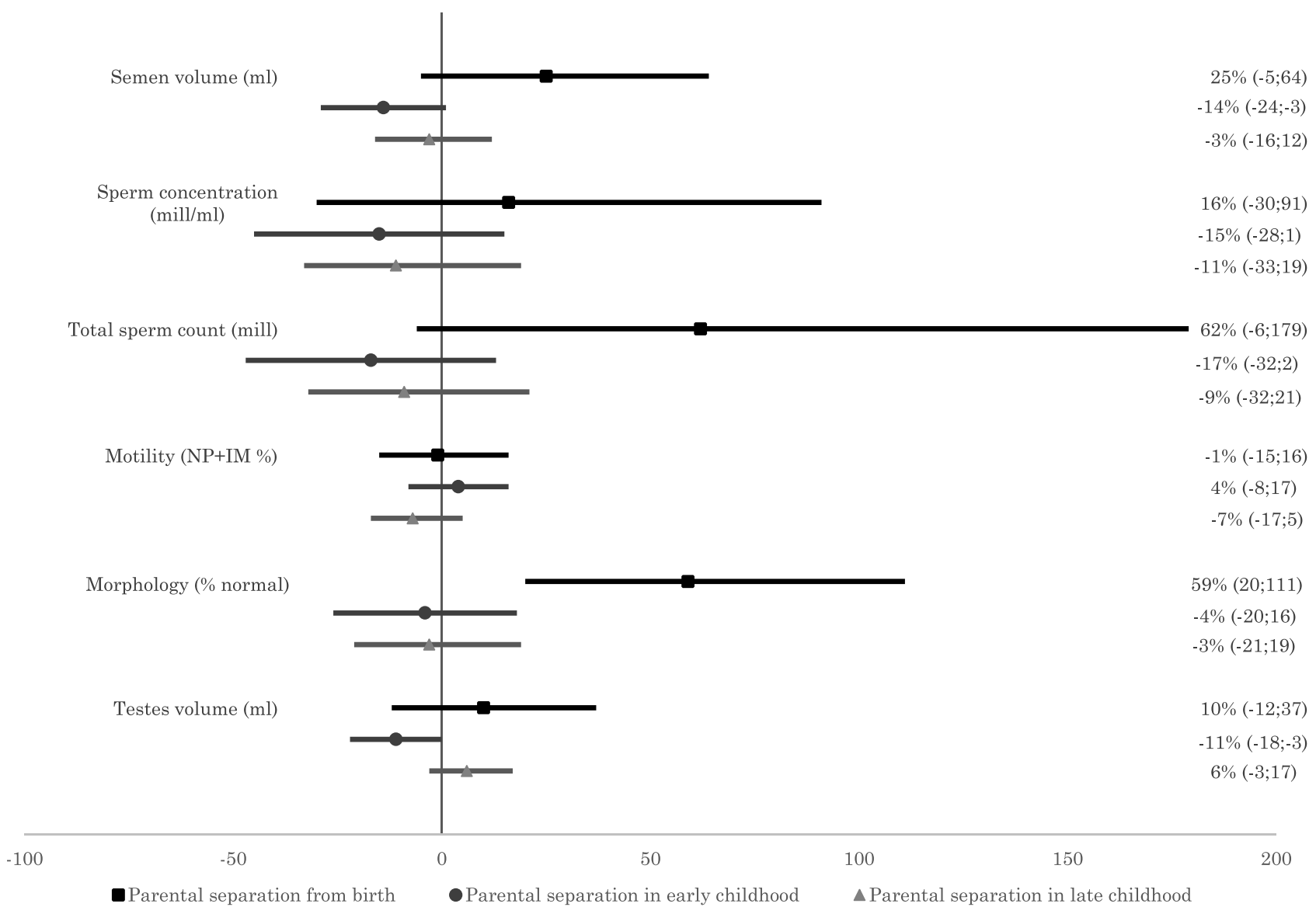

Figure 3 Main results exploring the timing of parental separation in childhood. Relative percentage differences in semen quality and testes volume according to timing of parental separation in childhood ( $\mathrm{N}=696)$. Parental separation from birth (square and black), in early childhood (circle and dark grey) or in late childhood (triangle and light grey) relatively to no parental separation (vertical line). Adjusted for maternal age at delivery; maternal social class; maternal first trimester smoking; abstinence time; place of semen sample collection (not testes volume); spillage (not testes volume). Motility further adjusted for interval between ejaculation and analysis in minutes. Due to model fit, the figure show results for NP (non-progressive) + IM (immotile) spermatozoa. Therefore, negative estimates represent a higher proportion of progressive motility, and positive estimates represent a lower proportion of progressive motility. Point estimates with $95 \%$ confidence intervals are shown to the right.

Information on parental separation in pregnancy was provided by the mothers in the first trimester interview. This may not apply to the entire pregnancy and in addition may reflect single motherhood and not parental separation. Thus, the parental separation in pregnancy exposure group may include women, who started cohabitation with their partner or spouse later in pregnancy, since only 10 sons reported parental separation from birth, together with women, who chose to establish a family without involving a father. However, less than five men were conceived using a sperm donor, and none of those were exposed to parental separation, either in pregnancy or in childhood.

Information on parental separation in childhood was provided by the children themselves in the 11-year follow-up. Most sons (83\%) completed the questions guided by a parent, limiting the risk of misclassifications. However, potential non-differential misclassification may arise in the categorized variable, especially around the introduced cut-offs between exposure groups. This could potentially mix the exposure groups and decrease exposure contrast, but we do not expect it to explain the observed associations.

Overall, information problems when measuring parental separation, semen characteristics and testes volume may give rise to potential information bias, which potentially could lead to bias towards the null.

The potential consequences for reproductive health of parental separation are likely to be modified by the level of family conflicts, shared parenting schedules, future family structures involving a potential stepfamily, potential loss of income, and lower socioeconomic status. ${ }^{16,19}$ We only measured parental separation and not the individual perceived stress. ${ }^{13}$ It may be more stressful for some children to live in a conflicted non-separated family environment than going through a parental separation, and therefore the stress levels may not be directly comparable. ${ }^{15}$ Furthermore, the 
consequences of parental separation are also likely to be modified by levels of societal support, making our results generalizable to other Western countries with high welfare schemes. ${ }^{23}$

\section{Interpretation}

Parental separation in pregnancy has to our knowledge never been investigated in relation to semen quality in adult sons as the primary focus. One previous study has investigated this as one of several prenatal stressful life events in one combined exposure group. ${ }^{12}$ The results suggested that maternal stressful life events in early pregnancy was associated with a lower total sperm count and lower progressive motility. In this smaller follow-up study, ${ }^{12}$ around $5 \%$ of the men were exposed due to parental separation or divorce in pregnancy, whereas, eg, death of a relative constituted the exposure for almost $10 \%$ of the men. The reported findings may therefore potentially reflect an association with more potent stressinducing events. First trimester maternal bereavement, assessed as the death of a relative, has previously been suggested to be associated with an increased risk of a composite measure of infertility, congenital malformations of genital organs, or testes cancer. However, when examining infertility in isolation, no associations were observed. ${ }^{11}$

Further, parental separation in childhood has to our knowledge never been investigated in relation to semen quality. However, in a study examining reproductive development following parental separation, we found that sons exposed to parental separation in childhood entered puberty earlier than their unexposed peers, ${ }^{26}$ indicating that parental separation may affect reproductive development. Moreover, the current study aligns with other research, suggesting that stressful life events in childhood may impair male reproductive health. ${ }^{10,22}$

Epigenetic mechanisms may offer a possible explanation for the observed associations. Epigenetic changes, in particular abnormal sperm DNA methylation, have been closely associated with impaired semen quality. ${ }^{37,38}$ Stress following parental separation in pregnancy might induce such epigenetic alterations. ${ }^{7,8}$

Telomere length is another potential biomarker of reproductive capability, since telomere shortening has been associated with impaired semen quality. ${ }^{39}$ Both prenatal stress and parental separation in childhood, father loss due to separation or death and childhood adversities have been associated with shortened telomere length. ${ }^{19,40-42}$ This offers a possible explanation for the observed associations between parental separation in childhood and semen quality in this study.

Though we had limited power, our results suggested that parental separation from birth might be associated with higher semen quality. Pregnancies in mothers of sons exposed to parental separation from birth were mainly unplanned $(80 \%)$. An unplanned pregnancy may be a result of a prompt conception likely to follow intercourse with a father with high or at least adequate semen quality, which may be inherited to the son. ${ }^{43,44}$ However, in the sub-analyses adjusting for unplanned pregnancy, the results did not change essentially, suggesting that our results should be explained by another unknown mechanism. However, the result may also be a chance finding, since only 10 participants were exposed to parental separation from birth.

\section{Conclusion}

Our results indicated that parental separation from birth was associated with higher semen quality and parental separation in early childhood was associated with lower semen quality in young men. Due to low statistical power, most confidence intervals included the null. However, the tendencies were evident for all semen quality characteristics, suggesting that the timing of parental separation in childhood was important for the associations with semen quality in this study. Since this is the first study investigating parental separation in relation to adult sons' semen quality and testes volume, our findings need replication.

\section{Abbreviations}

BMI, body mass index; CI, confidence interval; DNBC, Danish National Birth Cohort; FEPOS, Fetal Programming of Semen quality cohort; HPA, hypothalamic-pituitary-adrenal; HPG, hypothalamic-pituitary-gonadal; IM, immotile; NP, non-progressive; PR, progressive; SD, standard deviation. 


\section{Data Sharing Statement}

The dataset analysed in the study is not publicly available due to national data security legislation on sensitive personal data. Researchers may apply for access to data from the DNBC. Please see https://www.dnbc.dk/data-available or write to dnbc-research@ssi.dk for additional information.

\section{Acknowledgments}

We are grateful to all participants and to biomedical laboratory technicians Marianne Lipka Flensborg and Joan Dideriksen for running the clinics and collecting data. We also thank Josefine Rahbæk Larsen for assisting with recruitment and data entry, Lone Fredslund and Inge Eisensee for data management, and Cecilia Tingsmark for doing the morphology analysis. The Danish National Birth Cohort was established with a significant grant from the Danish National Research Foundation. Additional support was obtained from the Danish Regional Committees, the Pharmacy Foundation, the Egmont Foundation, the March of Dimes Birth Defects Foundation, the Health Foundation and other minor grants. The DNBC Biobank has been supported by the Novo Nordisk Foundation and the Lundbeck Foundation. Follow-up of mothers and children have been supported by the Danish Medical Research Council (SSVF 0646, 271-08-0839/06-066023, O602-01042B, 0602-02738B), the Lundbeck Foundation (195/04, R100-A9193), the Innovation Fund Denmark 0603-00294B (09-067124), the Nordea Foundation (02-2013-2014), Aarhus Ideas (AU R9-A959-13-S804), University of Copenhagen Strategic Grant (IFSV 2012), and the Danish Council for Independent Research (DFF - 4183-00594 and DFF - 4183-00152).

\section{Author Contributions}

A.G-S. performed data management in close collaboration with K.K.H. A.G.S. planned the study, performed the statistical analyses, and wrote the first draft in close collaboration with N.B. G.T. T.B.H. and C.H.R-H. S.S.T. and J.P. E.B acquired the funding, and G.T, S.S.T., K.K.H., K.S.H., J.P.E.B. and C.H.R-H. planned and headed the data collection for FEPOS. All authors contributed to data analysis, drafting or revising the article, have agreed on the journal to which the article will be submitted, gave final approval of the version to be published, and agree to be accountable for all aspects of the work.

\section{Funding}

This study is part of the ReproUnion collaborative study, co-financed by the European Union, Intereg V ÖKS (20200407). The FEPOS project was further funded by the Lundbeck Foundation (R170-2014-855), the Capital Region of Denmark, Medical doctor Sofus Carl Emil Friis and spouse Olga Doris Friis's Grant, Axel Muusfeldt's Foundation (2016-491), A.P. Møller Foundation (16-37), the Health Foundation and Dagmar Marshall's Fond. In addition, this study was supported by Aarhus University and Independent Research Fund Denmark (9039-00128B).

\section{Disclosure}

Dr Cecilia Høst Ramlau-Hansen reports grants from Independent research fund Denmark, during the conduct of the study. The authors report no other conflicts of interest in this work.

\section{References}

1. Levine H, Jorgensen N, Martino-Andrade A, et al. Temporal trends in sperm count: a systematic review and meta-regression analysis. Hum Reprod Update. 2017;23(6):646-659. doi:10.1093/humupd/dmx022

2. McSwiggin HM, O’Doherty AM. Epigenetic reprogramming during spermatogenesis and male factor infertility. Reproduction. 2018;156(2):R9-R21. doi:10.1530/REP-18-0009

3. Skakkebaek NE, Rajpert-de Meyts E, Buck Louis GM, et al. Male reproductive disorders and fertility trends: influences of environment and genetic susceptibility. Physiol Rev. 2016;96(1):55-97. doi:10.1152/physrev.00017.2015

4. Skakkebæk NE, Rajpert-de Meyts E, Main KM. Testicular dysgenesis syndrome: an increasingly common developmental disorder with environmental aspects: opinion. Human Reprod. 2001;16(5):972-978. doi:10.1093/humrep/16.5.972

5. Dada R, Kumar M, Jesudasan R, Fernández JL, Gosálvez J, Agarwal A. Epigenetics and its role in male infertility. Sci Reprod Med. 2012;29 (3):213-223.

6. Rajender S, Avery K, Agarwal A. Epigenetics, spermatogenesis and male infertility. Mutat Res. 2011;727(3):62-71. doi:10.1016/j.mrrev.2011.04.002

7. Glover V, Capron L. Prenatal parenting. Curr Opin Psychol. 2017;15:66-70. doi:10.1016/j.copsyc.2017.02.007 
8. Glover V. Prenatal stress and its effects on the fetus and the child: possible underlying biological mechanisms. In: Antonelli MC, editor. Perinatal Programming of Neurodevelopment. New York: Springer New York; 2015:269-283.

9. Barrett ES, Swan SH. Stress and androgen activity during fetal development. Endocrinology. 2015;156(10):3435-3441. doi:10.1210/en.2015-1335

10. Nargund VH. Effects of psychological stress on male fertility. Nat Rev Urol. 2015;12(7):373-382. doi:10.1038/nrurol.2015.112

11. Plana-Ripoll O, Li J, Kesmodel US, Parner E, Olsen J, Basso O. Reproductive function in the sons of women who experienced stress due to bereavement before and during pregnancy: a nationwide population-based cohort study. Fertil Steril. 2017;107(1):189-197.e188. doi:10.1016/j. fertnstert.2016.10.016

12. Brauner EV, Hansen AM, Doherty DA, et al. The association between in-utero exposure to stressful life events during pregnancy and male reproductive function in a cohort of 20-year-old offspring: the Raine study. Hum Reprod. 2019;34(7):1345-1355. doi:10.1093/humrep/dez070

13. Fink G. Stress: Concepts, Cognition, Emotion, and Behavior: Handbook of Stress Series. Vol. 1. San Diego, United States: Elsevier Science \& Technology; 2016.

14. Paul RA. The consequences of divorce for adults and children. J Marriage Fam. 2000;62(4):1269-1287. doi:10.1111/j.1741-3737.2000.01269.x

15. Troxel WM, Matthews KA. What are the costs of marital conflict and dissolution to children's physical health? Clin Child Fam Psychol Rev. 2004;7 (1):29-57. doi:10.1023/B:CCFP.0000020191.73542.b0

16. Thomas JR, Högnäs RS. The effect of parental divorce on the health of adult children. Longit Life Course Stud. 2015;6(3):279-302. doi:10.14301/ llcs.v6i3.267

17. Lansford JE. Parental divorce and children's adjustment. Perspect Psychol Sci. 2009;4(2):140-152. doi:10.1111/j.1745-6924.2009.01114.x

18. Nunes-Costa RA, Lamela DJ, Figueiredo BF. Psychosocial adjustment and physical health in children of divorce. J Pediatr (Rio J). 2009;85 (5):385-396. doi:10.2223/JPED.1925

19. Vezzetti VC. New approaches to divorce with children: a problem of public health. Health Psychol Open. 2016;3(2):2055102916678105 doi:10.1177/2055102916678105

20. Bloch M, Peleg I, Koren D, Aner H, Klein E. Long-term effects of early parental loss due to divorce on the HPA axis. Horm Behav. 2007;51 (4):516-523. doi:10.1016/j.yhbeh.2007.01.009

21. Toufexis D, Rivarola MA, Lara H, Viau V. Stress and the reproductive axis. J Neuroendocrinol. 2014;26(9):573-586. doi:10.1111/jne.12179

22. Ilacqua A, Izzo G, Emerenziani GP, Baldari C, Aversa A. Lifestyle and fertility: the influence of stress and quality of life on male fertility. Reprod Biol Endocrinol. 2018;16(1):115. doi:10.1186/s12958-018-0436-9

23. European Commission. Employment, social affairs \& inclusion: Denmark; 2020. Available from: https://ec.europa.eu/social/main.jsp?catId= 1107\&langId=en. Accessed June 10, 2021.

24. Keglberg Hærvig K, Bonde JP, Ramlau-Hansen CH, et al. Fetal programming of semen quality (FEPOS) cohort - a DNBC male-offspring cohort. Clin Epidemiol. 2020;12:757-770. doi:10.2147/CLEP.S242631

25. Olsen J, Melbye M, Olsen SF, et al. The Danish National Birth Cohort-its background, structure and aim. Scand J Public Health. 2001;29 (4):300-307. doi:10.1177/14034948010290040201

26. Gaml-Sørensen A, Brix N, Ernst A, Lunddorf LLH, Ramlau-Hansen CH. Father absence in pregnancy or during childhood and pubertal development in girls and boys: a population-based cohort study. Child Dev. 2021;92:1494-1508. doi:10.1111/cdev.13488

27. World Health Organization. WHO Laboratory Manual for the Examination and Processing of Human Semen. 5th ed. Geneva: World Health Organization; 2010.

28. Ramlau-Hansen CH, Thulstrup AM, Bonde JP, Ernst E. Is self-measuring of testicular volume by a Prader orchidometer a valid method? Fertil Steril. 2007;87(6):1480-1482. doi:10.1016/j.fertnstert.2006.11.032

29. Greenland S, Pearl J, Robins JM. Causal diagrams for epidemiologic research. Epidemiology. 1999;10(1):37-48. doi:10.1097/00001648199901000-00008

30. Bliddal M, Broe A, Pottegård A, Olsen J, Langhoff-Roos J. The Danish medical birth register. Eur J Epidemiol. 2018;33(1):27-36. doi:10.1007/ s10654-018-0356-1

31. Tang D, Kong D, Pan W, Wang L. Outcome model free causal inference with ultra-high dimensional covariates. 2020.

32. Kirkwood BR, Sterne JAC. Essential Medical Statistics. 2th ed. Malden, Mass: Blackwell Science; 2012.

33. Hernan MA, Hernandez-Diaz S, Robins JM. A structural approach to selection bias. Epidemiology. 2004;15(5):615-625. doi:10.1097/01. ede. 0000135174.63482 .43

34. Stewart TM, Liu DY, Garrett C, Brown EH, Baker HW. Recruitment bias in studies of semen and other factors affecting pregnancy rates in fertile men. Hum Reprod. 2009;24(10):2401-2408. doi:10.1093/humrep/dep215

35. Stokes-Riner A, Thurston SW, Brazil C, et al. One semen sample or 2? Insights from a study of fertile men. J Androl. 2007;28(5):638-643. doi:10.2164/jandrol.107.002741

36. Hart RJ, Doherty DA, McLachlan RI, et al. Testicular function in a birth cohort of young men. Hum Reprod. 2015;30(12):2713-2724. doi:10.1093/ humrep/dev244

37. Gunes S, Esteves SC. Role of genetics and epigenetics in male infertility. Andrologia. 2020;53:e13586. doi:10.1111/and.13586

38. Cui X, Jing X, Wu X, et al. DNA methylation in spermatogenesis and male infertility. Exp Ther Med. 2016;12(4):1973-1979. doi:10.3892/ etm.2016.3569

39. Thilagavathi J, Venkatesh S, Dada R. Telomere length in reproduction. Andrologia. 2013;45(5):289-304. doi:10.1111/and.12008

40. Marchetto NM, Glynn RA, Ferry ML, et al. Prenatal stress and newborn telomere length. Am J Obstet Gynecol. 2016;215(1):94.e91-94.e98. doi:10.1016/j.ajog.2016.01.177

41. Entringer S, Epel ES, Kumsta R, et al. Stress exposure in intrauterine life is associated with shorter telomere length in young adulthood. Proc Natl Acad Sci USA. 2011;108(33):E513-518. doi:10.1073/pnas.1107759108

42. Mitchell C, McLanahan S, Schneper L, Garfinkel I, Brooks-Gunn J, Notterman D. Father loss and child telomere length. Pediatrics. 2017;140(2). doi:10.1542/peds.2016-3245

43. Storgaard L, Bonde JP, Ernst E, et al. Genetic and environmental correlates of semen quality: a twin study. Epidemiology. 2006;17(6):674-681. doi:10.1097/01.ede.0000239730.47963.4e

44. Ferlin A, Raicu F, Gatta V, Zuccarello D, Palka G, Foresta C. Male infertility: role of genetic background. Reprod Biomed. 2007;14(6):734-745. doi:10.1016/S1472-6483(10)60677-3 


\section{Publish your work in this journal}

Clinical Epidemiology is an international, peer-reviewed, open access, online journal focusing on disease and drug epidemiology, identification of risk factors and screening procedures to develop optimal preventative initiatives and programs. Specific topics include: diagnosis, prognosis, treatment, screening, prevention, risk factor modification, systematic reviews, risk \& safety of medical interventions, epidemiology \& biostatistical methods, and evaluation of guidelines, translational medicine, health policies \& economic evaluations. The manuscript management system is completely online and includes a very quick and fair peer-review system, which is all easy to use.

Submit your manuscript here: https://www.dovepress.com/clinical-epidemiology-journal 